\title{
Access to Child Health Care, Medical Treatment of Sick Children and Childhood Mortality Relationships in Kenya
}

\author{
Boniface Omuga K'Oyugi \\ Population Studies and Research Institute, University of Nairobi, Nairobi, Kenya \\ Email: bkoyugi@uonbi.ac.ke
}

Received 26 March 2014; revised 29 April 2014; accepted 7 May 2014

Copyright (C) 2014 by author and Scientific Research Publishing Inc.

This work is licensed under the Creative Commons Attribution International License (CC BY). http://creativecommons.org/licenses/by/4.0/

(c) (i) Open Access

\begin{abstract}
Child health care factors such as medical treatment of sick children have direct and indirect effect on childhood mortality. Through international cooperation, a number of countries including Kenya have gathered information on provision of child health services at facility level from periodic Service Provision Assessment (SPA) surveys. Kenya has also gathered information on medical treatment of sick children at household level from periodic Demographic and Health Surveys (DHS). However, establishing how health care information in the SPA surveys relate to childhood mortality and also how these factors relate to medical treatment of sick children in the DHS has been constrained by differences in sample designs of the surveys. This study deployed a strategy of constructing community level variables derived from the SPA survey data and incorporated them into DHS data which served as the main data source. The SPA and DHS sampling designs for Kenya allow computation of stable estimates of regional demographic and health service indicators at provincial level. This study analyzed information gathered from 690 health facilities in 2010 SPA and 6079 births born less than 60 months from 2008/09 DHS. The study found that access to child health services, waiting time before service in facility and time to the nearest referral facility were significant facilitating factors for medical treatment of sick children. The study also established that waiting time before service in facility was the only access to health care factor which had significant effect on childhood mortality when HIV prevalence was excluded in the analysis. However, the significance of waiting time before service diminished with inclusion of HIV prevalence. Further research is required to refine definition and measurement for child health care variable on female autonomy.
\end{abstract}

\section{Keywords}

Access to Child Health Care, Medical Treatment, Childhood Mortality, Kenya 


\section{Introduction}

Access to child health care is an important component of quality health service provision. Medical treatment of sick children and other child health care factors are also critical determinants of childhood mortality according to the Mosley and Chen framework for analysis of child survival in developing countries [1]. In Kenya, one out of every 14 children die before attaining age five years and the causes of morbidity responsible for the deaths are mainly preventable diseases such as acute respiratory infections, malaria, anemia and malnutrition [2]. The estimate of under-five mortality rate from 2008/09 Kenya Demographic and Health Survey (KDHS) was 74 per 1000 live births. This rate was considered unacceptably very high giving that Kenya's target for the year 2015 is 32 per 1000 live births. To accelerate the pace of childhood mortality reduction, the Government of Kenya has been implementing a number of strategic interventions including: rolling out the Integrated Management of Childhood Illnesses (IMCI); the Child Health Promotion which involves immunization; and, Malaria Control. The IMCI strategy is aimed at ensuring that health facilities have: enough drugs and supplies; skilled providers; and, family and community components, among others. Due to sample design issues limiting combined use of KDHS and Kenya Service Provision Assessment (KSPA) surveys' data which are collected after every five years, establishing how access to child health care factors including medical treatment of sick children relates with childhood mortality in Kenya has remained as a major challenge. This study attempted to tackle the challenge by combined use of KDHS and KSPA data but confining the use of KSPA data to computations of provincial regional level estimates for child health services variables.

Indicators and measurements for access to child health services and medical treatment of sick children in Kenya are contained in the 2010 KSPA Report and the 2008/09 KDHS Report, respectively. At national level 2010 KSPA Report contained the following relevant key highlights that: 1) basic child health services were available in 68 percent of the facilities; 2) all first-line medicines to treat diarrhea, malaria and emergency conditions defined in IMCI strategy were available in 66 percent of facilities offering outpatient care for sick children; 3) reasons given by mothers as to why they failed to seek health care from the nearest facility included waiting time before being attended to and shortage of prescribed medicines [3]. The national level 2008/09 KDHS Report relevant key highlights included: 1) only 56 percent of sick children who were reported to have suffered from acute respiratory infections within two weeks from the survey date in the household received medical treatment; 2) only 49 percent of the children who had diarrhea were taken to health facility for medical treatment [4]. The two reports also provide provincial regional level estimates for these key indicators and hence underscore the existence of wide variations. Kenya is therefore far from achieving universal access to health care services.

This study benefited greatly from a comprehensive systematic review of studies undertaken in sub-Saharan Africa by Rutherford, Mulholland and Hill on how access to health care relates to under-five mortality [5]. The review study concluded that there was a need to include in analysis of other measures such as social networks, time available to access health care and female autonomy in addition to traditional measures of distance from health services and cost of health care access. Results of literature review undertaken for this study, also made the following conclusions: 1 ) death in children aged under five years is strongly associated with adequate health care whereby 41 to 72 percent of new born deaths can be avoided through coverage of current health care interventions [6]; and, 2) implementation of modern child health care programmes was responsible for reductions in child deaths in sub-Saharan countries such as Liberia, Zaire, Senegal and Mozambique [7]-[9]. Some of the studies undertaken using Kenyan data concluded as follows: 1) that heath care delivery system pose recurrent barriers to the accessing of health care for the under-five and by implication frustrate efforts towards childhood mortality reduction [10]; and, 2) efforts of unmeasured environmental and community factors such as availability of hospitals and economic resources are likely to be important for child mortality [11].

\section{Data and Methods}

\subsection{Data}

The data on child health care variables used in this study were obtained from the KSPA survey conducted in 2010. This was the second in the series of health facility surveys undertaken in Kenya usually about one year after the KDH surveys. The 2010 KSPA covered 690 health facilities carefully sampled to represent the distribution of a total of 6192 functional health facilities at the time of the survey in Kenya. The sample took into consideration the following key background characteristics: 1) type of facility (hospital, health center, maternity, 
clinic and dispensary); 2) managing authority (government, non-governmental organization, private for profit and faith based organization); 3) administrative province (Nairobi, Central, Coast, Eastern, North Eastern, Nyanza, Rift Valley and Western). The instruments used in the assessment were developed based on best practices and lessons learnt from past health facility assessments implemented in a number of countries including Kenya. In addition, the survey used extensively guidelines and standards developed by the World Health Organization (WHO) technical programmes and the work of the International Health Facility Assessment Network (IHFAN). Details of the service readiness indicators' definition, data collection and references are contained in the 2012 WHO publication [12]. In addition, the 2010 KSPA Survey Report contains detailed descriptions of survey process, methodology including the criteria for selection of the sample and data collection instruments [3].

Given the focus of this study, the following specific information on child health services obtained from the 2010 KSPA survey were utilized: 1) availability of child health services at the facility with a bias on curative outpatient care for sick children, growth monitoring and childhood immunization; 2) capacity at the facility to provide quality outpatient care for sick children with bias on essential medicine and supplies as spelt out in the IMCI strategy and relevant health provider pre or in-service training undertaken within 12 months prior to the survey; 3) waiting time before service and other client satisfaction information from exit interviews of sampled sick children caretakers and service providers; and, 4) availability of services for prevention of mother-to-child transmission (PMTCT) of HIV with a bias on facility capacity to provide minimum package of PMTCT and having all items of PMTCT plus services. This was a special consideration based on established fact from previous studies that HIV/AIDS was mainly responsible for upsurge of child mortality rate in the period 1998-2003 [13].

The data on medical treatment of sick children and other control variables used in the study were obtained from the 2008/09 KDHS. Detailed descriptions of the survey process, methodology and data collection instruments are contained in the 2008/09 KDHS Report [2]. Since information on exact dates of births and dates of interviews in the households were available in the KDHS data set, the analysis was restricted to 6079 births born under 60 months prior to the survey date in their respective households. The inherent constraint regarding use of two data sets with different sample designs was resolved by: 1) restricting the use of 2010 KSPA data to computation of community/contextual variables at provincial level consistent with the shared sample domain with the 2008/09 KDHS; and, 2) assuming that the health facility conditions had not changed substantially between 2009 and 2010.

\subsection{Variables in the Analytical Framework and Methods}

The choice of variables included in this analysis was guided by the Mosley-Chen (1984) conceptual framework on proximate determinants [1]. Its basic assumption is that child mortality is as a result of the background variables operating through proximate determinants or by proximate determinants directly. Some of the important proximate variables include: access to and use of child health services; exposure and susceptibility to varying pathogenic agents; and, nutritional status. The proximate factors are also assumed to depend upon social and economic factors such as parental education and household disposable income and wealth. Fortunately for this study, a number of studies on determinants of child mortality applying Mosley-Chen framework have been undertaken using Kenyan data which have resulted into determination of statistically significant factors and variable categorization based on empirical relative risk of child mortality. This study took cognizance that in Kenya, child mortality had been adversely affected directly and indirectly by HIV/AIDS epidemic. Variables selection was therefore guided by literature on child mortality determinants in Kenya and in sub-Sahara Africa. The analysis was restricted to only 7 variables on access to child health care which included medical treatment of sick children. Additional 10 other variables were also included in the analysis as controls.

In view of the limitations of the 2008/09 KDHS data as appertains to quality of data on use of child health services at household level, it became necessary to compute weighted aggregated values for these variables at district level since cluster and household codes in the data set had been scrambled prior to release of data for public use in order to guard against revealing identifiers of individuals who provided HIV/AIDS information. Similarly, the nature of the 2010 KSPA dataset only allowed for computation of weighted aggregated values of variables on child health services at provincial level due to its sampling design. All the child health care variables were therefore treated as contextual/community level variables in this study. This was a major limitation 
since wide variations also exist at household level as appertains to access to child health services. However, it is worth noting that the results of this study have low probability of adverse effects associated with use of mixed methodology of qualitative and quantitative data collection simply because the implementation of both the KSPA and KDHS surveys in Kenya received technical support from the ICF Macro and were part of the world wide surveys conducted after rigorous pilot evaluations of WHO recommended survey designs and methodologies including data collection instruments to enhance accuracy and international comparability of results.

The study used deaths in the six age segments of childhood as the outcome variable. During the preliminary stages in the analysis, a total of 17 independent variables that were considered for inclusion were: 1) 4 community/contextual variables used as controls (mean length of breastfeeding duration, full immunization coverage rate, malaria severity and HIV prevalence rate); 2) 7 variables on access to and use of child health services (availability of child health services, waiting time before service in facility, time to the nearest referral facility, child health services satisfaction level, female autonomy on health care with special emphasis on financial and empowerment for seeking health care, medical treatment of sick children and availability of PMTCT services); 3 socio-economic variables used as controls (wealth index, maternal marital status and mother's education level); and, 3 proximate determinant variables also used as controls (single/multiple birth, sex and toilet facility type). A 60 and 40 percentile rule was applied across the board to classify all values of the independent variables, based on perceived risk of childhood death, into "better-off” and "worse-off" categories respectively.

Generalized linear regression model in log-linear format was used to undertake bivariate and multivariate analysis. Detailed exposition on the application of the model in analysis of childhood mortality in Kenya can be obtained from the 2014 study by K’Oyugi [14]. Equation (1) presents the model in a mathematical expression [15].

$$
\log \left(\theta_{x}\right)=\beta_{0}+\beta_{1} x_{1}+\beta_{2} x_{2}+\cdots+\beta_{p} x_{p}
$$

where:

$\theta_{x}=E(T \mid x)$ and a more functional form that is easy to use is $\theta_{x}=\exp (X \beta)$;

$X=\left(x_{1} \cdots x_{p}\right)$ vector of regression variables;

$\beta=\left(\beta_{1} \cdots \beta_{p}\right)$ vector of regression coefficients;

$E$ = expectation or mean of the statistical distribution;

$T=$ life time exponentially distributed;

$t=$ failure time;

$p=$ number of regression variables;

$\beta_{0}$ is the intercept term.

Cross tabulation method was also applied in bivariate analysis to determine the nature of relationships between the variable on medical treatment of sick children and each of the other child health care variables. In addition, life table method was used extensively to compute childhood mortality rates so as to establish childhood mortality differential by various independent categorical variables analyzed.

\section{Results and Discussion}

The results of this study are presented and discussed in three sub-sections namely: childhood mortality age patterns of access to child health care factors; medical treatment of sick children and other child health care factors linkages; and, effects of child health care and medical treatment of sick children on childhood mortality in Kenya. In discussing the obtained results, efforts were made to gauge their consistency with the study expectations based on massive literature that accompanied the description of the 1984 Mosley and Chen analytical framework for analysis of child survival in developing countries plus results from some of similar studies already highlighted [1] [5] [13] [14].

\subsection{Childhood Mortality Age Patterns of Access to Child Health Care Factors}

The childhood mortality age patterns of the already listed seven variables on access to child health care were analyzed. As stated earlier, the choice of the variables was based on most recent literature on how child care access relates to child mortality especially in sub-Saharan Africa. Brief descriptions of computation procedures for each of the seven variables are provided in this sub-section. The obtained age specific childhood mortality rates for each variable by category are presented in Table 1. 
Table 1. Childhood mortality rates in Kenya by access to child health care factors.

\begin{tabular}{|c|c|c|c|c|c|c|}
\hline \multirow{2}{*}{ Variable/Category } & \multicolumn{6}{|c|}{ Probability of dying by exact age in months (per 1000 live births) } \\
\hline & 1 & 3 & 6 & 12 & 24 & 60 \\
\hline \multicolumn{7}{|c|}{ Access to child health services } \\
\hline Better & 0.025 & 0.032 & 0.039 & 0.049 & 0.063 & 0.078 \\
\hline Poorer & 0.041 & 0.047 & 0.052 & 0.061 & 0.067 & 0.072 \\
\hline \multicolumn{7}{|c|}{ Child health services satisfaction level } \\
\hline High & 0.033 & 0.039 & 0.044 & 0.050 & 0.057 & 0.065 \\
\hline Low & 0.025 & 0.032 & 0.041 & 0.052 & 0.070 & 0.086 \\
\hline \multicolumn{7}{|c|}{ Waiting time before service in facility } \\
\hline Shorter & 0.026 & 0.033 & 0.038 & 0.045 & 0.053 & 0.062 \\
\hline Longer & 0.035 & 0.041 & 0.051 & 0.065 & 0.087 & 0.107 \\
\hline \multicolumn{7}{|c|}{ Time to nearest referral facility } \\
\hline Shorter & 0.029 & 0.036 & 0.040 & 0.049 & 0.058 & 0.065 \\
\hline Longer & 0.028 & 0.034 & 0.045 & 0.055 & 0.074 & 0.094 \\
\hline \multicolumn{7}{|c|}{ Female autonomy on health care } \\
\hline More empowered & 0.029 & 0.035 & 0.042 & 0.052 & 0.064 & 0.073 \\
\hline Less empowered & 0.029 & 0.035 & 0.042 & 0.051 & 0.064 & 0.081 \\
\hline \multicolumn{7}{|c|}{ Access to PMTCT services } \\
\hline Better & 0.030 & 0.037 & 0.046 & 0.056 & 0.072 & 0.087 \\
\hline Poorer & 0.027 & 0.033 & 0.038 & 0.047 & 0.056 & 0.066 \\
\hline \multicolumn{7}{|c|}{ Medical treatment of sick children } \\
\hline Higher & 0.029 & 0.034 & 0.040 & 0.049 & 0.062 & 0.073 \\
\hline Lower & 0.029 & 0.037 & 0.044 & 0.054 & 0.066 & 0.079 \\
\hline National average & 0.028 & 0.035 & 0.042 & 0.051 & 0.064 & 0.076 \\
\hline
\end{tabular}

\subsubsection{Access to Child Health Services}

This variable was constructed from information gathered in the 2010 KSPA survey. It consists of three indicators namely: percentage of facilities offering all the basic three child health services (curative outpatient care, growth monitoring and childhood immunization); percentage of facilities offering sick child services having essential medicines and supplies that meet the requirements specified in the IMCI strategy; and, percentage of facilities with child health service providers reported receiving routine training related to child health as specified in the IMCI strategy. Each broader community as used in this study was then classified to belong to either "better-off" or "worse-off" category based on values for each of the three indicators constituting the variable. The overall variable categorization was achieved by applying the 60 - 40 percentile rule on the computed indicators' values distribution. In this study, communities with at least two of the three indicators in the better-off category was classified as having better access to child health services and those with less than 2 of the indicators in the worse-off category were classified as having poorer access to child health services. This study results suggest that access to child health care services were relatively better in Nairobi, Eastern, Nyanza, Rift Valley, Western and North Eastern provinces. The results also classified Central and Coast provinces as having poorer access to child health services.

This study's expectation was that communities with better access to child health services should have lower childhood mortality rates when compared to those with poorer access. The life table analysis results on age patterns of childhood mortality presented in Table 1 were therefore found to be consistent with the expectation except at age 60 months. However, the difference was considered not substantial.

\subsubsection{Child Health Services Satisfaction Level}

The study used responses from facility client exit interviews in the 2010 KSPA survey. The survey sought the opinion of clients (care takers) who had brought sick children to facility on their satisfaction using a structured 
instrument that had listed 13 aspects of possible problem areas with respect to service delivery as contained in the guidelines formulated by WHO and adopted by Kenya. The aspects included quality of examination, availability of medicine and health provider treatment of client, among others. A weighted distribution of mean number of total aspects of problems listed by clients in each community was computed and by applying the 60 - 40 percentiles rule, communities with average of 4 or less listed aspects of problem were classified as being more satisfied and those with 5 or more listed aspects were classified as being less satisfied. The computed variable suggests that child health services satisfaction level was relatively higher in Central, Coast, Eastern and North Eastern provinces. The results also suggest that satisfaction level was relatively lower in Nairobi, Nyanza, Rift Valley and Western provinces.

It was the expectation in this study that communities with higher child health satisfaction level should have relatively lower childhood mortality rates when compared to their counterparts with lower satisfaction level. The childhood mortality age patterns obtained from life table analysis presented in Table 1 were found not consistent with the expectation at all the ages. This was therefore a result requiring further research that was outside the scope of this study.

\subsubsection{Waiting Time before Service in Facility}

Client exit interview information on waiting time before service in the 2010 KSPA survey was used to compute this variable. Weighted mean durations of waiting time in minutes at community level were computed and the 60 - 40 percentile rule applied on the obtained values of the distribution to facilitate categorization. Communities with more than 58 minutes of waiting time before service were placed in the longer category and those with 58 minutes or less were placed in the shorter category. In this regard, Coast, Eastern, North Eastern, Rift Valley and western provinces fell in the category shorter waiting time while Nairobi, Central and Nyanza provinces were in the longer waiting time category.

Life table analysis of childhood mortality age patterns for this variable in Table 1 was found to be consistent with the study expectation whereby those with shorter waiting time had lower mortality rates at all ages.

\subsubsection{Time to the Nearest Referral Facility}

This variable served as proxy for time to service facility which was not captured in the 2010 KSPA survey. This information was obtained from service provider interviews in facility. Weighted mean durations of time in minutes to the nearest referral facility at community level were computed and the 60 - 40 rule applied on the obtained values to facilitate classification. In this study, communities with travel time exceeding 62 minutes were considered to have longer duration while those 62 or less minutes were considered to have shorter duration. The results suggests that Nairobi, Central Coast, Rift Valley and Western provinces had relatively shorter travel time to referral facility while Eastern, North Eastern and Nyanza provinces had longer travel time to referral facility. It was this study expectation that communities with shorter travel time to referral facility should have lower childhood mortality rates. The results in Table 1 were therefore generally consistent with the expectation except at ages 1 and 3 months. However, the observed differences were considered not substantial.

\subsubsection{Female Autonomy on Health Care}

A proxy variable was computed from two indicators using information gathered in the 2008/09 KDHS. The survey had sought information from individual women interviewed on who had the final say on their own health care and on their type earnings from work. In this study, those who reported to have the final say alone or jointly with their partners and in addition reported to be earning some cash for work were classified as belonging to more empowered category while the rest were classified as less empowered. To stabilize the community level estimated values, weighting and aggregation of data was done at district level. The 60 - 40 percentiles rule was applied to the distribution of obtained weighted values on percentages of female empowerment thus classifying communities with over 41 percent as being more empowered and those with 41 percent or less as being less empowered. The expectation of this study was that communities with relatively higher female autonomy on health care should have lower childhood mortality rates. Life table results in Table 1 showed no or small differences except at age 60 months. However, the general pattern was found to be consistent with the expectation.

\subsubsection{Access to PMTCT Services}

This variable was computed from two indicators derived from the 2010 KSPA survey. The survey gathered in- 
formation on: percentage of facilities offering minimum package of PMTCT and having all items of PMTCT plus; and, percentage of facilities with PMTCT providers trained within three years prior to the survey. The 60 40 percentile rule was applied to the distribution of weighted values for each of the two indicators. The variable access to PMTCT services was then computed using the criteria of those communities with over 33 percent of facilities offering the services and with over 72 percent of facilities having trained PMTCT service providers as belonging to the better category. Those communities not meeting the threshold were classifies as belonging to the poorer category. The criteria placed Coast, Nyanza and Rift valley provinces in the category of better access to PMTCT services while Nairobi and Central provinces were placed in the category of poorer access to PMTCT services. The expectation of this study was that communities with better access to PMTCT services should have lower childhood mortality rates. The life table analysis results in Table 1 indicated that this expectation was not met at all ages. This result also required further scrutiny which was outside the scope of this study.

\subsubsection{Medical Treatment of Sick Children}

This variable was computed directly using information gathered in the 2008/09 KDHS on if children who had been reported to have had diarrhea or acute respiratory infection within two weeks prior to the survey received medical treatment from recognized sources recommended in the IMCI strategy. To cater for the large number of cases without this information in the households, weighted percentages of children who received medical treatment at district level were computed. The 60 - 40 percentile rule was applied to the distribution of obtained values whereby those with more than 46 percent were classified as belonging to the higher category and those with 46 percent or less were classified as belonging to lower category. It was the expectation of this study that communities with higher percentages of medical treatment of sick children should have lower childhood mortality rates. The results of the life table analysis in Table 1 were consistent with the expectation at all ages.

\subsection{Medical Treatment of Sick Children and Other Child Health Care Factors Linkages}

In the Mosley-Chen analytical model for child survival in developing countries, the variable medical treatment of sick children is one of the child health care factors and hence operates at the same level. Establishing the relationships between medical treatment of sick children and each of the other six health care variables analyzed was necessary given that it was to be retained in the analysis to the final stage due to its study importance. Since the other health care variables in the analytical model were conceptualized be serving as facilitating variables which assist in influencing whether or not sick children would be provided with medical treatment, it was therefore the expectation in this study that each of the health care variables would have significant association with medical treatment of sick children. It was therefore expected that those in worse-off category in each other child health care variables, based on perceived higher risk of childhood mortality, would mainly be in the category with worse-off medical treatment of sick children and the vice versa. Simple cross tabulation and correlation analysis were undertaken to establish direction and strength of the relationships. Since the worse-off category for the variable medical treatment of sick children variable was coded 2 and strategically the worse-off categories for the other health care variables were also coded 2, no positive correlation coefficients between medical treatment of sick children and each of the other health care variables were expected. The results of the analysis provided in Table 2 indicated that only three out of the six other health care variables met the expectation. This could be interpreted to mean that the study data only confirmed that access to child health services, waiting time before service in the facility and time to nearest referral facility served as significant facilitative factors to medical treatment of sick children.

\subsection{Effects of Child Health Care Factors and Medical Treatment of Sick Children on Childhood Mortality in Kenya}

\subsubsection{Results of Bivariate Regression}

As part of the screening process aimed at establishing which of the seven health care variables included in this study had statistically significant effect on childhood mortality, separate bivariate regression models were undertaken. In each model, age interval variable was included to cater for the age pattern of childhood mortality while deaths in the age interval and logarithm of exposures in the age interval served as the regression outcome variable and off-set variable respectively. The results are provided in Table 3. 
Table 2. Association between medical treatment of sick children and other child health care factors.

\begin{tabular}{cccccccc}
\hline \multirow{2}{*}{ Medical treatment of sick children and } & \multicolumn{3}{c}{ Cross tabulation results } & \multicolumn{3}{c}{ Correlation analysis results } \\
\cline { 2 - 7 } & $\chi^{2}$ & df & Sig. & R & T & Sig. \\
\hline Access to child health services & 293.066 & 1 & $0.000^{* * * *}$ & -0.220 & -17.544 & $0.000^{* * * *}$ \\
Child health services satisfaction level & 324.563 & 1 & $0.000^{* * * *}$ & 0.231 & 18.514 & $0.000^{* * *}$ \\
Waiting time before service in facility & 86.636 & 1 & $0.000^{* * *}$ & -0.119 & -9.373 & $0.000^{* * *}$ \\
Time to nearest referral facility & 20.724 & 1 & $0.000^{* * *}$ & -0.058 & -4.559 & $0.000^{* * *}$ \\
Female autonomy on health care & 56.121 & 1 & $0.000^{* * * *}$ & 0.096 & 7.526 & $0.000^{* * *}$ \\
Access to PMTCT services & 40.146 & 1 & $0.000^{* * *}$ & 0.082 & 6.435 & $0.000^{* * *}$ \\
\hline
\end{tabular}

Key: Sig.—Significance; df—degrees of freedom; $\chi^{2}$ —Pearson’s Chi Square; R—Pearson's Correlation Coefficient; T—Student T statistic; ${ }^{* * *}$ - significant at $99 \%$ confidence level.

Table 3. Parameter estimates of bivariate regression models on effects of child health care factors on childhood mortality.

\begin{tabular}{|c|c|c|c|c|c|c|}
\hline \multirow{2}{*}{ Variable/Category } & \multicolumn{6}{|c|}{ Regression model fitness } \\
\hline & $\beta$ & SE & Sig. & $\chi^{2}$ & df. & Sig. \\
\hline Access to child health services & & & & 0.352 & 1 & 0.553 \\
\hline Better & 0.075 & 0.1272 & 0.553 & & & \\
\hline \multicolumn{7}{|l|}{ Poorer $[\mathrm{RC}]$} \\
\hline Sick child caretaker service satisfaction level & & & & 1.092 & 1 & 0.296 \\
\hline High & -0.114 & 0.1094 & 0.296 & & & \\
\hline \multicolumn{7}{|l|}{ Low $[\mathrm{RC}]$} \\
\hline Waiting time before service in facility & & & & 6.964 & 1 & $0.008^{* * *}$ \\
\hline Shorter & -0.282 & 0.1067 & $0.008^{* * *}$ & & & \\
\hline \multicolumn{7}{|l|}{ Longer $[\mathrm{RC}]$} \\
\hline Time to nearest referral facility & & & & 4.998 & 1 & $0.025^{* *}$ \\
\hline Shorter & -0.237 & 0.1059 & $0.025^{* *}$ & & & \\
\hline \multicolumn{7}{|l|}{ Longer $[\mathrm{RC}]$} \\
\hline Female autonomy on health care & & & & 0.524 & 1 & 0.469 \\
\hline More empowered & 0.079 & 0.1094 & 0,469 & & & \\
\hline \multicolumn{7}{|l|}{ Less empowered $[\mathrm{RC}]$} \\
\hline Access to PMTCT Services & & & & 0.378 & 1 & 0.539 \\
\hline Better & 0.067 & 0.1082 & 0.539 & & & \\
\hline \multicolumn{7}{|l|}{ Poorer $[\mathrm{RC}]$} \\
\hline Medical treatment of sick children & & & & 2.877 & 1 & $0.090^{*}$ \\
\hline Higher & 0.184 & 0.1085 & $0.090^{*}$ & & & \\
\hline Lower $[\mathrm{RC}]$ & & & & & & \\
\hline
\end{tabular}

Key: RC—Reference Category; $\beta$ —Regression coefficient; SE—Standard Error; Sig.—Significance; df.—degrees of freedom; —-significant at $90 \%$ confidence level; ${ }^{* *}$ — significant at $95 \%$ confidence level; ${ }^{* * *}$ — significant at $99 \%$ confidence level.

The bivariate regression results indicate that only two health care variables included namely waiting time before service at facility and time to the nearest referral facility had statistically significant reduction effects on childhood mortality at 99 and 95 percent confidence levels, respectively. The child health care variable of medical treatment of sick children though having statistically significant effect on childhood mortality at 90 percent confidence level, had unexpected direction of effect. Child health services satisfaction level variable was found to have statistically insignificant reduction effect on childhood mortality. As for the remaining three health care variables namely access to child health services, female autonomy on health care and access to PMTCT services, the results indicate that they were statistically highly insignificant with unexpected direction of influence. This could simply be interpreted to mean that the data did not support the study expectations on these three variables. 


\subsubsection{Results of Multivariate Regression}

Results of bivariate analysis involving the health care and other control variables included in this study were used in fitting of the multivariate models ran. On the part of the health care variables, waiting time to before service at facility and time to the nearest referral facility were included in the multivariate models on the strength of the bivariate analysis results. Medical treatment of sick children was also included in the multivariate models due to its importance to the study objectives. As justified earlier in Section 2.1, the following 10 control variables were considered for inclusion in the multivariate models: 4 community level variables (mean length of breastfeeding duration, full immunization coverage rate, malaria severity and HIV prevalence rate); 3 socioeconomic variables (wealth index, marital status and mother's education level); and 3 proximate determinants (type of birth, sex and type of floor material).

Each of the control variables were subjected first to bivariate level regression and then to sub-model screening prior to inclusion in the full multivariate regression models used to determine the effects of health care factors on childhood mortality. The bivariate screening established that all the control variables except malaria severity and sex had statistically significant reduction effect on childhood mortality. The sub-model screening process was able to eliminate malaria severity and time to the nearest facility variables on the basis of their very high correlation coefficients with the other variables in the models.

The three final multivariate regression models and whose parameter estimates are provided in Table 4 were fitted being guided by the 1984 Mosley-Chen analytical framework with the sole objective of determining the effects of child health care factors on childhood mortality. Model I included the control variables in the analysis but excluded the child health care variables. Putting into consideration of the Hill, Bacigo and Mahy milestone study finding to the effect that HIV/AIDS was the single most important factor explaining the 1998-2003 upsurge in child mortality in Kenya [13], the strategy adopted in this study was to fit Model II which included the child health care variables and control variables without HIV prevalence rate. Model III was then fitted including the child health care variables and control variables with HIV prevalence rate.

Estimates of the parameters for goodness of fit of the 4 multivariate regression models used to study the effects of child health care factors are also provided in Table 4. Their statistical tests of significance were based on their computed Likelihood ratio $\chi^{2}$ values and as indicated in the table all the 4 models were found to be highly statistically significant at 99 percent level of confidence.

Results of Model I established that when child health care variables are not taken into consideration, five variables namely age interval, HIV prevalence rate, wealth index, marital status and type of birth are significant factors affecting childhood mortality in Kenya. The situation changed considerably in Model II which had child health care variables and all other control variables except HIV prevalence rate. Waiting time before service became the only child health care variable that was found to have statistically significant reduction effect on childhood mortality at 99 percent confidence level. In this regard, those births in communities with relatively shorter waiting time experienced about 31 percent reduction in childhood mortality when compared to their other counterparts. The other health care variable namely medical treatment of sick children in Model II was found to have statistically insignificant effect and also had unexpected direction. In addition, five control variables in Model II were found to be significant determinants of childhood mortality at 90 percent confidence level or higher. These significant control variables were: age interval, full breastfeeding coverage rate, maternal marital status, mother's education level and type of birth.

Results of Model III which had child health care variables plus control variables including HIV prevalence rate, established that the child health care variable waiting time before service become statistically insignificant. However, shorter waiting before service was still found to have about 19 percent reduction effect on childhood mortality relative to their other counterparts. The other health care variable namely medical treatment of sick children in Model III was found to be statistically insignificant with unexpected direction of effect as was in Model II. In addition, the Model III parameter estimates also established that five control variables had statistically significant reduction effects on childhood mortality. The five variables were: age interval; HIV prevalence rate; maternal marital status; and, mother's education level.

\section{Conclusions and Recommendations}

The major conclusions and recommendations of the study are presented in three sub-sections in conformity with the three broad areas analyzed namely: age patterns of childhood mortality by child health care factors; medical 
Table 4. Parameter estimates of impact of child health care, medical treatment of sick children and control factors on infant and child mortality in Kenya.

\begin{tabular}{cccccccccccc}
\hline \multirow{2}{*}{ Parameter } & \multicolumn{4}{c}{ Model I } & \multicolumn{4}{c}{ Model II } & \multicolumn{3}{c}{ Model III } \\
\cline { 2 - 11 } & $\boldsymbol{\beta}$ & SE & Sig. & $\boldsymbol{\beta}$ & SE & Sig. & $\boldsymbol{\beta}$ & SE & Sig. \\
\hline (Intercept) & 1.576 & 0.251 & $0.000^{* * *}$ & 1.139 & 0.258 & $0.000^{* * *}$ & 1.124 & 0.256 & $0.000^{* * *}$
\end{tabular}

\section{Age interval (in months)}

$\begin{array}{cccccccccc}\text { 1-under 3 } & -1.907 & 0.180 & 0.000^{* * *} & -1.567 & 0.177 & 0.000^{* * *} & -1.584 & 0.175 & 0.000^{* * *} \\ \text { 3-under 6 } & -2.193 & 0.177 & 0.000^{* * *} & -1.769 & 0.174 & 0.000^{* * *} & -1.779 & 0.173 & 0.000^{* * *} \\ \text { 6- under 12 } & -2.798 & 0.164 & 0.000^{* * *} & -2.164 & 0.161 & 0.000^{* * *} & -2.171 & 0.160 & 0.000^{* * *} \\ \text { 12-under 24 } & -3.184 & 0.154 & 0.000^{* * *} & -2.323 & 0.152 & 0.000^{* * *} & -2.336 & 0.151 & 0.000^{* * *} \\ \text { 24-under 60 } & -4.936 & 0.236 & 0.000^{* * *} & -3.959 & 0.230 & 0.000^{* * *} & -3.964 & 0.228 & 0.000^{* * *} \\ \text { Under1 [RC] } & 0.000 & . & . & 0.000 & . & . & 0.000 & . & .\end{array}$

Mean length of breastfeeding duration

$\begin{array}{cccccccccc}\text { Longer } & -0.085 & 0.105 & 0.415 & -0.113 & 0.103 & 0.273 & -0.104 & 0.102 & 0.307 \\ \text { Shorter [RC] } & 0.000 & . & . & 0.000 & . & . & 0.000 & . & \text {. }\end{array}$

Full immunization coverage rate

$\begin{array}{cccccccccc}\text { Higher } & -0.061 & 0.110 & 0.579 & -0.210 & 0.103 & 0.043^{* *} & -0.115 & 0.113 & 0.309 \\ \text { Lower [RC] } & 0.000 & . & . & 0.000 & . & . & 0.000 & . & \\ \text {. }\end{array}$

HIV prevalence rate

$\begin{array}{cccc}\text { Lower } & -0.450 & 0.110 & 0.000^{* *} \\ \text { Higher [RC] } & 0.000 & . & .\end{array}$

Medical treatment of sick children

$$
\text { Higher }
$$

Lower [RC]

\section{Waiting time before service in facility}

Shorter
Longer [RC]

Wealth index (quintile)

$\begin{array}{cccccccccc}3^{\text {rd }} & 0.258 & 0.140 & 0.066^{*} & -0.183 & 0.137 & 0.183 & -0.193 & 0.136 & 0.155 \\ 4^{\text {th }} \text { and } 5^{\text {th }} & 0.044 & 0.140 & 0.752 & -0.182 & 0.128 & 0.156 & -0.184 & 0.127 & 0.148 \\ 1^{\text {st }} \text { and } 2^{\text {nd }} & 0.000 & & & 0.000 & & & 0.000 & & \end{array}$

Marital status

\begin{tabular}{|c|c|c|c|c|c|c|c|c|c|}
\hline Married & -1.035 & 0.313 & $0.001^{* * *}$ & -1.127 & 0.306 & $0.000^{* * *}$ & -1.136 & 0.304 & $0.000^{* * *}$ \\
\hline Never & -0.894 & 0.154 & $0.000^{* * *}$ & -0.219 & 0.151 & 0.147 & -0.211 & 0.150 & 0.159 \\
\hline \multicolumn{4}{|c|}{ Widow/divorced/separated [RC] } & 0.000 & & & 0.000 & & \\
\hline \multicolumn{10}{|c|}{ ther' education level } \\
\hline Secondary ${ }^{+}$ & -0.065 & 0.131 & 0.618 & -0.244 & 0.127 & $0.056^{*}$ & -0.249 & 0.126 & $0.048^{* *}$ \\
\hline imary complete & -0.192 & 0.153 & 0.208 & -0.246 & 0.142 & $0.084^{*}$ & -0.274 & 0.141 & $0.052^{*}$ \\
\hline \multicolumn{4}{|c|}{ one/primary incomp. [RC] } & 0.000 & & & 0.000 & & \\
\hline
\end{tabular}

\section{Type of birth}

\begin{tabular}{ccccccccccc} 
Single & -1.302 & 0.177 & $0.000^{* * *}$ & -0.332 & 0.177 & $0.060^{* *}$ & -0.324 & 0.175 & $0.064^{*}$ \\
Multiple [RC] & 0.000 &. &. & 0.000 &. &. & 0.000 &. &. \\
\hline
\end{tabular}




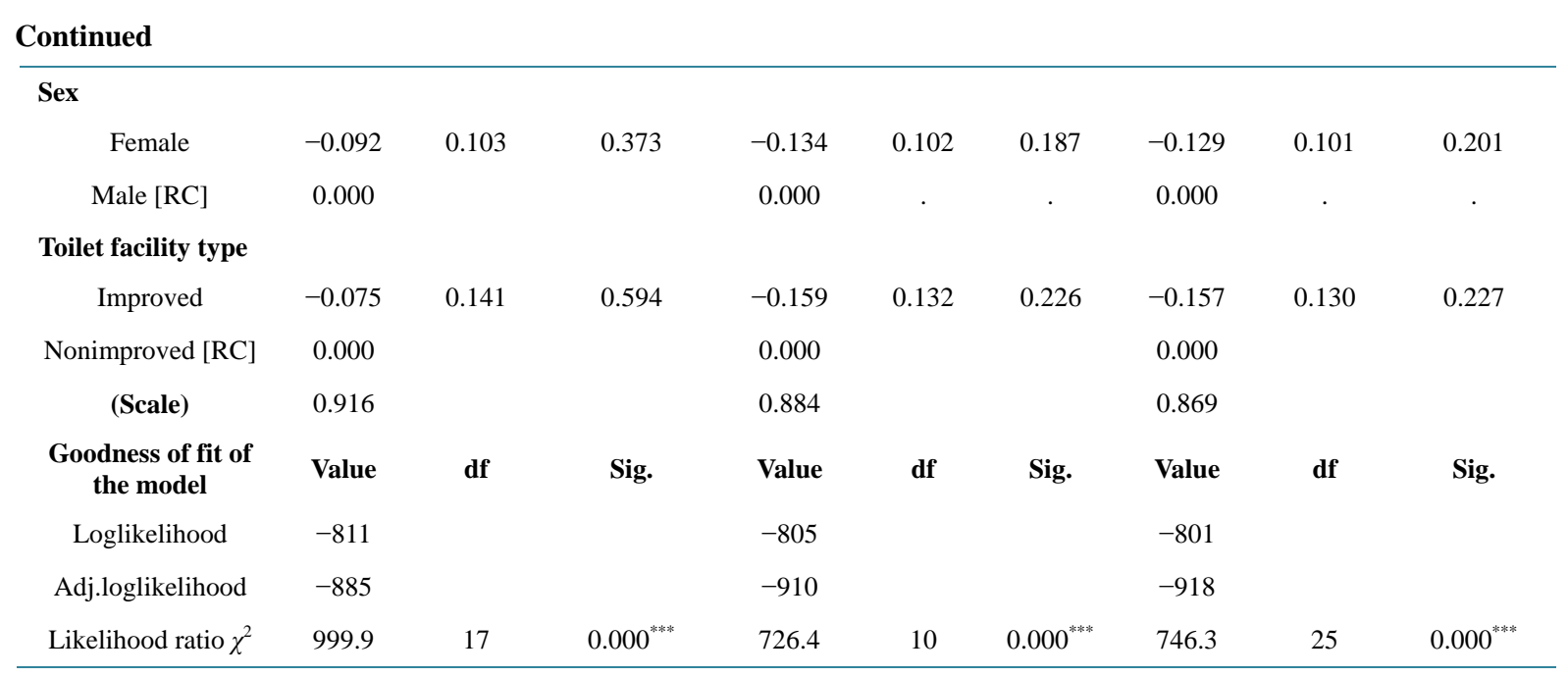

Key: RC—Reference Category; $\beta$-Regression coefficient; SE—Standard Error; Sig.—Significance; df—degrees of freedom; ${ }^{*}$ - significant at $90 \%$ confidence level; ${ }^{* *}$ — significant at $95 \%$ confidence level; ${ }^{* * *}$ — significant at $99 \%$ confidence level.

treatment of sick children and other child health care factors linkages; and, effects of child health care on childhood mortality.

\subsection{Age Patterns of Childhood Mortality}

This study established that all the 7 health care variables analyzed except 2 had age patterns that were consistent with the study expectations. The two exceptions were child health services satisfaction level and access to PMTCT services. In addition the study data suggest that female autonomy as a health care variable although yielded consistent age patterns with the study expectation, had no substantial differences in childhood mortality rates between those births belonging to more empowered females and of less empowered except at age 60 months.

The study findings on age patterns imply that deliberate efforts should be intensified in the existing child survival programmes in Kenya towards improving further level of child health services satisfaction in facility. It should also be noted that both the United Nations Human Rights Charter and the 2010 Constitution of Kenya require that the country must provide quality health services to all its citizens including children. The tolerance limit of 4 or less listed aspects of problem areas applied in this study was far above the expected zero value. Kenya is still far from attaining the child health services standards as stipulated in the WHO guidelines which were adopted by the Ministry of Health. In addition, there is a need for further research to: 1) unpack child health services satisfaction level so as to isolate region specific aspects of problem areas to facilitate strengthening child survival programmes in Kenya; and, 2) refine measurements and indicators for female autonomy variable beyond use of who has final saying on health care and cash earnings for work by females in households.

\subsection{Medical Treatment of Sick Children and Other Child Health Care Factors}

The major finding of this study on this particular aspect was that improved medical treatment had statistically significant association with improved access to child health services, shorter waiting time before service in facility and shorter time to the nearest referral facility. This could be interpreted to mean that desired further improvements in level of medical treatment of sick children could be achieved by mainly improving the indicators constituting the variable access which included: availability of facilities; child health services provider skills, and, stock of essential medicine and supplies in facility.

\subsection{Effects of Child Health Care on Childhood Mortality}

At the bivariate level, this study established that shorter waiting time before service in facility and shorter time to the nearest referral facility had significant reduction effects on childhood mortality. The multivariate analysis established that: 1) without HIV prevalence rate, shorter waiting time before service in facility had about 31 
percent statistically significant reduction effect on childhood mortality relative to their counterparts belonging to the category longer waiting time; and, 2) with HIV prevalence rate in the model, waiting time before service in facility was found to have statistically insignificant reduction effect on childhood mortality estimated at about 19 percent lower for those in shorter waiting time category relative to their other counterparts.

The findings on effect of child health care on childhood mortality amplify strongly the need for minimizing delay to reach facility and to provide services in facility. The results also underscore the importance of waiting time before service in facility as a critical health care factor in childhood mortality reduction. Further reduction of HIV prevalence rate is critical since lower rates were found to have statistically significant beneficial impact on childhood mortality and were also found to capture the effect of waiting time for child health services in facility.

\section{Ethical Approval}

The study used copies of the following two data files which are available for research purposes only: 1) the KDHS 2008-09 from the Kenya National Bureau of Statistics; and, 2) the KSPA 2010 from the National Council for Population and Development. The Scientific Research Committee of the Kenya Medical Research Institute a State Corporation of the Government of Kenya gave ethical clearance for the undertaking of the two surveys.

\section{References}

[1] Mosley, W.H. and Chen, L. (1984) An Analytical Framework for the Study of Child Survival in Developing Countries. Population and Development Review, 10, 25-45. http://dx.doi.org/10.2307/2807954

[2] National Council for Population and Development (2012) A Look at the Child Health Services in Kenya. Service Provision Assessment-Child Health Services, NCPD Policy Brief No. 24, 1-5.

[3] National Coordinating Agency for Population and Development and ICF Macro (2011) Kenya Service Provision Assessment 2010. NCAPD and ICF Macro, Nairobi, 62-168.

[4] Kenya National Bureau of Statistics and ICF Macro (2010) Kenya Demographic and Health Survey 2008-09. KNBS and ICF Macro, Calverton, 132-137.

[5] Rutherford, M.E., Mulholland, K. and Hill, P. (2010) How Access to Health Care Relates to Under-Five Mortality in Sub-Saharan Africa: Systematic Review. Tropical Medicine and International Health, 15, 508-519. http://dx.doi.org/10.1111/j.1365-3156.2010.02497.x

[6] Haines, A., Sanders, D., Lehmann, U., Rowe, A.K., Lawn, J.E., Jan, S., et al. (2007) Achieving Child Survival Goals: Potential Contribution of Community Health Workers. Lancet, 369, 2121-2131. http://dx.doi.org/10.1016/S0140-6736(07)60325-0

[7] Ewbank, D.C. (1993) Impact of Health Programmes on Child Mortality in Africa: Evidence from Zaire and Liberia. International Journal of Epidemiology, 22, S64-S72. http://dx.doi.org/10.1093/ije/22.Supplement 1.S64

[8] Poison, G., Trape, J., Lefebure, M. and Enel, C. (1993) Rapid Decline in Child Mortality in a Rural Area of Senegal. International Journal of Epidemiology, 22, 72-80. http://dx.doi.org/10.1093/ije/22.1.72

[9] Cutts, F., Dos Santos, C., Novoa, A., David, P., Macassa, G. and Soares, A. (1996) Child and Maternal Mortality during a Period of Conflict in Beria City, Mozambique. International Journal of Epidemiology, 25, 349-356. http://dx.doi.org/10.1093/ije/25.2.349

[10] Opwora, A.S., Laving, A.M.R., Nyabola, L.O. and Olenja, J.M. (2011) Who Is to Blame? Perspectives of Caregivers on Barriers to Accessing Healthcare for the Under-Fives in Butere District, Western Kenya. BMC Public Health, 11, 272. http://dx.doi.org/10.1186/1471-2458-11-272

[11] Omariba, D.W.R., Beaujot, R. and Rajulton, F. (2007) Determinants of Infant and Child Mortality in Kenya: An Analysis Controlling for Frailty Effects. Population Research and Policy Review, 26, 299-321. http://dx.doi.org/10.1007/s11113-007-9031-z

[12] World Health Organization (2012) Measuring Service Availability and Readiness Assessment. World Health Organization, Geneva, 1-30.

[13] Hill, K., Bicego, G. and Mahy, M. (2001) Childhood Mortality in Kenya: An Examination of Trends and Determinants in the Late 1980s to Mid1990s. Working Paper 01-01, John Hopkins University, Hopkins Population Centre, Baltimore.

[14] K’Oyugi, B.O. (2014) Impact of Breastfeeding on Infant and Child Mortality with Varying Levels of Immunization and HIV/AIDS: Evidence from 2008/09 Kenya Demographic and Health Survey. Social Sciences, 3, 9-16. 
[15] Breslow, N.E. and Day, N.E. (1987) Statistical Methods in Cancer Research Volume II-The Design and Analysis of Cohort Studies. IARC Scientific Publications No. 82, International Agency for Research on Cancer, Lyon, 120-162.

\section{Abbreviations}

AIDS: Acquired Immune Deficiency Syndrome

DHS: Demographic and Health Survey

IHFAN: International Health Facility Assessment Network

IMCI: Integrated Management of Childhood Illnesses

HIV: Human Immunodeficiency Virus

KDHS: Kenya Demographic and Health Survey

KSPA: Kenya Service Provision Assessment

PMTCT: Prevention of Mother to Child Transmission

WHO: World Health Organization 